TRANSACTIONS OF THE

AMERICAN MATHEMATICAL SOCIETY

Volume 358, Number 7, Pages 3219-3237

S 0002-9947(06)03851-7

Article electronically published on February 20, 2006

\title{
EFFECTIVE CONES OF QUOTIENTS OF MODULI SPACES OF STABLE $n$-POINTED CURVES OF GENUS ZERO
}

\author{
WILLIAM F. RULLA
}

\begin{abstract}
Let $X_{n}:=\bar{M}_{0, n}$, the moduli space of $n$-pointed stable genus zero curves, and let $X_{n, m}$ be the quotient of $X_{n}$ by the action of $\mathcal{S}_{n-m}$ on the last $n-m$ marked points. The cones of effective divisors $\overline{N E}^{1}\left(X_{n, m}\right), m=0,1,2$, are calculated. Using this, upper bounds for the cones $\operatorname{Mov}\left(X_{n, m}\right)$ generated by divisors with moving linear systems are calculated, $m=0,1$, along with the induced bounds on the cones of ample divisors of $\bar{M}_{g}$ and $\bar{M}_{g, 1}$. As an application, the cone $\overline{N E}^{1}\left(\bar{M}_{2,1}\right)$ is analyzed in detail.
\end{abstract}

\section{INTRODUCTION}

In studying an object $X$ in the category of projective varieties, it is useful to know to which other objects $X$ admits surjective morphisms, or more generally, dominant rational maps. Thus, of interest are the sets of line bundles which have basepoint free, or more generally, non-empty, linear series. The closures $\operatorname{Nef}^{1}(X)$ and $\overline{N E}^{1}(X)$ of the corresponding cones in the real vector space $N^{1}(X)$ can sometimes be determined with nothing more than basic intersection theory.

The intermediate cone $N e f^{1}(X) \subseteq M o v(X) \subseteq \overline{N E}^{1}(X)$, generated by all linear series $|M|$ which have base loci bl $|M|$ of codimension $\geq 2$, is of greater interest than $\overline{N E}^{1}(X)$ in studying rational maps $\varphi_{|D|}: X \rightarrow Z$, since it is natural to extend a map $\varphi_{|D|}$ over a Cartier divisor $E \in \mathrm{bl}|D|$ by considering $|M:=D-E|$ instead. $\operatorname{Mov}(X)$ similarly has an upper bound $\operatorname{Nem}(X)$ which is potentially calculable via basic intersection theory. The purpose of this paper is to introduce the "nem" cone and some of its properties, and calculate it for certain finite quotients of the moduli spaces $\bar{M}_{0, n}$ of stable $n$-pointed curves of genus zero.

Until recently it was conjectured that the cones $\overline{N E}^{1}\left(\bar{M}_{0, n}\right)$ were generated by the irreducible components of the loci of singular curves $\Delta \subseteq \bar{M}_{0, n}$. This is now known to be false for $n \geq 6$ ([HT02] or [Ver02]), but the analogous statement is true for the quotients $X_{n, 0}:=\bar{M}_{0, n} / \mathcal{S}_{n}$ by the natural action of the symmetric group. A natural question (S. Keel) is: for which $m$ does the analogous statement hold for

Received by the editors December 5, 2003 and, in revised form, September 9, 2004.

2000 Mathematics Subject Classification. Primary 14E05, 14H10; Secondary 14E30.

Key words and phrases. Moduli space, rational curve, birational geometry, classification of morphisms/rational maps.

This paper is a product of a VIGRE seminar on $\bar{M}_{0, n}$ conducted by V. Alexeev at the University of Georgia, Athens, during the Spring of 2002. Thanks to S. Keel for posing the question motivating the paper, and to him, R. Varley, and E. Izadi for help and advice. Thanks also to the referee for many valuable comments. PORTA was used in calculating several examples. Xfig was used for the figures.

(C)2006 American Mathematical Society 3219

Reverts to public domain 28 years from publication 
the quotients $X_{n, m}:=\bar{M}_{0, n} / \mathcal{S}_{n-m}$, where $\mathcal{S}_{n-m}$ acts on the last $n-m$ marked points? This paper provides the answer: for $m \leq 2$ or $n \leq 5$ (Propositions 4.2, 5.3. and (7.2). Also included is a systematic treatment and extension of some of the ad hoc calculations of [Rul01], relating moduli spaces $\bar{M}_{g, k}$ to various $X_{n, m}$. It is the author's intent to use these results for ongoing work on $\bar{M}_{3}$ and other spaces.

The paper is structured as follows: $\$ 2$ establishes notation, definitions, and basic properties of $\mathrm{Nem}(X)$.

In $₫ 3$ bases for the real vector spaces $N^{1}\left(X_{n, m}\right)(0 \leq m \leq 3)$ are calculated.

44 pulls back the counterexample of [HT02] and Ver02] in $X_{6,3}$ to all $X_{n, m}$, $n \geq 6$ and $m \geq 3$.

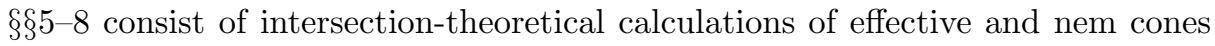
and examples.

In $₫ 9$ the calculations of $N e m\left(X_{2 g+2,0}\right)$ and $N e m\left(X_{2 g+3,1}\right)$ are used to impose bounds on the cones of ample divisors of $\bar{M}_{g}$ and $\bar{M}_{g, 1}$, respectively, via surjections of these spaces onto the corresponding loci of hyperelliptic curves.

As an application, in $\$ 10$ the cone of effective divisors $\overline{N E}^{1}\left(\bar{M}_{2,1}\right)$ of the moduli space $\bar{M}_{2,1}$ of stable genus 2 curves with marked point is studied in detail.

\section{Notation, DEFinitions, AND FUnCTORIAL PROPERTIES}

Notation. Let $X$ be a projective variety, over a(n algebraically closed) field $k$. The characteristic $c=$ char $k$ will be arbitrary, except for the restrictions $c \neq 2$ in $\S 99$ and 10, and $c=0$ for the applications of Mori theory in $\$ 10$

(1) The description "QQQ-factorial" will imply normality.

(2) The symbol "E" will be used to denote numerical equivalence (of Cartier divisors or one-cycles).

(3) $N_{1}(X)$ is the group of one-cycles modulo numerical equivalence, with real coefficients Kol96, p. 122].

(4) $N^{1}(X)$ is the group of Cartier divisors modulo numerical equivalence, with real coefficients [Kol96, p. 123].

(5) The cone of effective divisors $\overline{N E}^{1}(X)$ is the closure of the cone in $N^{1}(X)$ generated by (classes of) line bundles having non-zero global sections.

(6) A $\mathbb{Q}$-Cartier divisor $M \in \overline{N E}^{1}(X)$ will be called "moving" if there is a positive integer $n$ such that ( $n M$ is Cartier and) the base locus of the linear series $|n M|$ is of codimension $\geq 2$. The cone of moving divisors $\operatorname{Mov}(X)$ is the closure of the subcone of $\overline{N E}^{1}(X)$ generated by moving divisors.

(7) The cone of nef divisors $N e f^{1}(X)$ is the closure of the subcone of $\overline{N E}^{1}(X)$ generated by line bundles having basepoint free linear systems. Following Miles Reid, we think of the word "nef" as an acronym for "numerically eventually free."

(8) The cone of nef curves $N e f_{1}(X)$ is the cone in $N_{1}(X)$ dual to $\overline{N E}^{1}(X)$.

Following Miles Reid,

Definition. Let $X$ be a projective variety. A $\mathbb{Q}$-Cartier divisor $M \in \overline{N E}^{1}(X)$ will be called "nem" (for "numerically eventually moving") if for every prime divisor $D \subseteq X,\left.M\right|_{D} \in \overline{N E}^{1}(D)$. The cone of nem divisors $N e m(X)$ is the closure of the cone in $\overline{N E}^{1}(X)$ generated by nem $\mathbb{Q}$-Cartier divisors. 
Remarks. (1) Clearly the nem cone is an upper bound on the moving cone:

$$
\operatorname{Mov}(X) \subseteq N e m(X) .
$$

(2) For surfaces, the nef, moving, and nem cones are the same.

(3) If $D \equiv \sum \alpha_{i} D_{i}$ where $\alpha_{i}>0$ and the $D_{i}$ are prime divisors, then $D \in$ $N e m(X)$ iff $\left.D\right|_{D_{i}} \in \overline{N E}^{1}\left(D_{i}\right)$ for each $i$.

(4) If $M \in N e m(X)$ is a prime divisor and $C \in N e f_{1}(M)$, then $C \in N e f_{1}(X)$.

We prove some functorial properties.

2.1. Proposition. If $f: X \rightarrow Y$ is a dominant, finite morphism of projective varieties, then $f^{*} \operatorname{Nem}(Y) \subseteq N e m(X)$. Thus also

$$
\operatorname{Mov}(Y) \subseteq N e m(Y) \subseteq f_{*} N e m(X) .
$$

Proof. Let $N \in N e m(Y)$ be an effective Cartier divisor. Pick an irreducible component $M_{0}$ of $f^{*} N$ and let $N_{0}:=f\left(M_{0}\right)$. Then $\left.\left(f^{*} N\right)\right|_{M_{0}}=\left(\left.f\right|_{M_{0}}\right)^{*}\left(\left.N\right|_{N_{0}}\right) \in$ $\overline{N E}^{1}\left(M_{0}\right)$.

2.2. Lemma. If $f: X \rightarrow Y$ is a dominant, generically finite morphism of $\mathbb{Q}$ factorial, projective varieties, then $\operatorname{Mov}(Y) \subseteq f_{*} \operatorname{Mov}(X)$.

Proof. If $N \in \operatorname{Mov}(Y)$ is a Cartier divisor with $|N|$ having base locus of codimension $\geq 2$, then every divisorial component of the base locus of $\left|f^{*} N\right|$ must be exceptional for $f$. We may then produce a moving line bundle $\tilde{N}$, mapping to (a multiple of) $N$, by subtracting these components.

2.3. Lemma. Let $f: X \rightarrow Y$ be a birational morphism of projective varieties with $Y$ normal. If a Cartier $M$ moves on $X$ and $f_{*} M$ is also Cartier, then $f_{*} M$ moves on $Y$.

Proof. Because $f$ has connected fibres, any divisorial component $B$ in the base locus of $\left|f_{*} M\right|$ would have strict transform contained in the base locus of $|M|$.

2.4. Proposition. Let $f: X \rightarrow Y$ be a birational morphism of projective, $\mathbb{Q}$ factorial varieties. Then

$$
\operatorname{Mov}(Y)=f_{*} \operatorname{Mov}(X) \subseteq f_{*} \operatorname{Nem}(X) .
$$

Remark. Proposition 2.4 holds when $f$ is only a "birational contraction" HK00, Def. 1.0], i.e. a birational map such that the exceptional locus of $f^{-1}$ is of codimension $\geq 2$.

Definitions. (1) For any $0 \leq m \leq n$, define $X_{n, m}:=\bar{M}_{0, n} / \mathcal{S}_{n-m}$, where $\mathcal{S}_{n-m}$ acts on the last $n-m$ marked points. We abbreviate $X_{n, n}=X_{n, n-1}$ by $X_{n}$.

(2) Consider the quotient map $\pi: X_{n} \rightarrow X_{n, m}$, which makes indistinguishable the last $n-m$ marked points. We call the first $m$ marked points of an element of $X_{n, m}$ the distinguished marked points.

(3) We call the collection of images of boundary divisors of $X_{n}$ the boundary divisors of $X_{n, m}$. We denote them by $D_{T}^{i}$, where $i$ refers to the total number of marked points on one component of a general member of the locus $(2 \leq i \leq n-2)$ and $T$ is the subset of $\{1, \ldots, m\}$ labeling the distinguished marked points on that component $(0 \leq \# T \leq \min \{m, n-2\})$. For $X_{n}$, we drop the superscript $i=\# T$. Note that the notation has some redundancy; e.g. in $X_{n}, D_{T}=D_{T^{c}}$. 


\section{The groups $N^{1}\left(X_{n, m}\right)$}

We determine bases for the groups $N^{1}\left(X_{n, m}\right)$ for $n \geq 5$ and $0 \leq m \leq 3$. From Kee92, the spaces $N^{1}\left(\bar{M}_{0, n}\right)$ are spanned by the boundary classes $D_{T}$, subject only to the relations $\left(D_{T}=D_{T^{c}}\right.$ and $)$

$$
\sum_{\substack{i, j \in T \\ k, l \notin T}} D_{T} \equiv \sum_{\substack{i, k \in T \\ j, l \notin T}} D_{T}, \quad \text { for distinct } i, j, k, l \in\{1, \ldots, n\} .
$$

3.1. Lemma. The $\left(\begin{array}{l}n \\ 2\end{array}\right)-n$ relations $(3 \leq i<j \leq n, 4 \leq k \leq n)$

$$
\sum_{\substack{1,2 \in T \\ i, j \notin T}} D_{T} \equiv \sum_{\substack{1, i \in T \\ 2, j \notin T}} D_{T} \quad \text { and } \quad \sum_{\substack{1,3 \in T \\ 2, k \notin T}} D_{T} \equiv \sum_{\substack{1, k \in T \\ 2,3 \notin T}} D_{T}
$$

form a basis for the kernel of the vector space homomorphism

$$
\bigoplus \mathbb{R} \cdot D_{T} \rightarrow N^{1}\left(\bar{M}_{0, n}\right)
$$

where the direct sum is over the set of all boundary divisors $D_{T}$ of $\bar{M}_{0, n}$.

Proof. The dimension of the kernel is $\left(\begin{array}{l}n \\ 2\end{array}\right)-n$ Kee92]; suppose there is a relation

$$
\sum_{3 \leq i<j \leq n} a_{i j}\left(\sum_{\substack{1,2 \in T \\ i, j \notin T}} D_{T}-\sum_{\substack{1, i \in T \\ 2, j \notin T}} D_{T}\right)+\sum_{k=4}^{n} b_{k}\left(\sum_{\substack{1,3 \in T \\ 2, k \notin T}} D_{T}-\sum_{\substack{1, k \in T \\ 2,3 \notin T}} D_{T}\right) \equiv 0 .
$$

Each of the terms $D_{\{1,2, \ldots, n\} \backslash\{i, j\}}$ appears exactly once, having $a_{i j}$ as its coefficient, so $a_{i j}=0$. In the remaining sum, each $D_{\{1,2, \ldots, n\} \backslash\{2, k\}}$ appears exactly once, with coefficient $b_{k}$, so $b_{k}=0$.

3.2. Corollary. - The boundary divisors of $X_{n, 0}$ and $X_{n, 1}$ are a basis for their respective groups $N^{1}$.

- The boundary divisors of $X_{n, 2}$ are subject to the single relation

$$
\sum_{i=2}^{n-2}(n-i)(n-i-1) D_{12}^{i} \equiv \sum_{i=2}^{n-2}(i-1)(n-i-1) D_{1}^{i} .
$$

- The boundary divisors of $X_{n, 3}$ are subject only to the following relations (which are independent for $n \geq 5$ ):

$$
\begin{aligned}
\sum_{i=2}^{n-2}(n-i-1) D_{12}^{i} & \equiv \sum_{i=2}^{n-2}(n-i-1) D_{13}^{i} \equiv \sum_{i=2}^{n-2}(n-i-1) D_{23}^{i} \quad \text { and } \\
\sum_{i=3}^{n-2}(n-i)(n-i-1) & D_{123}^{i}+\sum_{i=2}^{n-3}(n-i-1)(n-i-2) D_{12}^{i} \\
& \equiv \sum_{i=3}^{n-2}(i-2)(n-i-1) D_{13}^{i}+\sum_{i=2}^{n-3}(i-1)(n-i-2) D_{1}^{i} .
\end{aligned}
$$

Proof. These statements follow from pushing down the relations (11) to the appropriate $N^{1}\left(X_{n, k}\right)$.

Corollary 3.2 is summarized in Table 1 
TABLe $1 . N^{1}\left(X_{n, k}\right), n \geq 5$

\begin{tabular}{||c|c|c||}
\hline & Picard number & \# of boundaries \\
\hline$X_{n, 0}$ & $\left\lfloor\frac{n}{2}\right\rfloor-1$ & $\left\lfloor\frac{n}{2}\right\rfloor-1$ \\
\hline$X_{n, 1}$ & $n-3$ & $n-3$ \\
\hline$X_{n, 2}$ & $2 n-7$ & $2 n-6$ \\
\hline$X_{n, 3}$ & $4 n-16$ & $4 n-13$ \\
\hline
\end{tabular}

\section{Counterexamples for $X_{n, m}, n \geq 6$ And $m \geq 3$}

4.1. Observation. For $n=6, \overline{N E}^{1}\left(X_{6,3}\right)$ is not generated by boundary classes.

Proof. From Corollary [3.2. $\rho\left(X_{6,3}\right)=8$, an ordered basis is given by $\left(D^{2}, D_{1}^{2}, D_{2}^{2}\right.$, $\left.D_{3}^{2}, D^{3}, D_{1}^{3}, D_{2}^{3}, D_{3}^{3}\right)$, and in terms of this basis,

$$
\text { and } \quad \begin{aligned}
3 D_{12}^{2} & \equiv(-1,1,1,0,-3,1,1,-1), \\
3 D_{13}^{2} & \equiv(-1,1,0,1,-3,1,-1,1), \\
3 D_{23}^{2} & \equiv(-1,0,1,1,-3,-1,1,1) .
\end{aligned}
$$

From [HT02] or Ver02 the class

$$
\begin{aligned}
F_{\tau} \equiv D_{36}+D_{46}+D_{56}-D_{16}-D_{26} \\
\quad-D_{136}-D_{146}-D_{236}-D_{246}+D_{346}+D_{356}+D_{12}
\end{aligned}
$$

lies in the effective cone of $X_{6}$, and (using (2) ) pushes down under the quotient morphism $X_{6} \rightarrow X_{6,3}$ (making indistinguishable the last three marked points) to

$$
\begin{aligned}
\pi_{*} F_{\tau} \equiv & 2 D_{3}^{2}+D^{2}+D^{2}-2 D_{1}^{2}-2 D_{2}^{2} \\
& \quad-2 D_{2}^{3}-2 D_{1}^{3}-2 D_{1}^{3}-2 D_{2}^{3}+2 D_{3}^{3}+2 D_{3}^{3}+6 D_{12}^{1} \\
= & 2(0,0,0,1,-3,-1,-1,1) .
\end{aligned}
$$

This is clearly not in the convex hull of the boundary divisors of $X_{6,3}$.

For $n \geq 6$, a basis for $N^{1}\left(X_{n, 3}\right)$ is given by \{boundary divisors $\} \backslash\left\{D_{12}^{2}, D_{13}^{2}\right.$, $\left.D_{23}^{2}\right\}$. In terms of an analogous ordered basis $\left(D^{2}, D_{1}^{2}, D_{2}^{2}, D_{3}^{2}, D^{3}, \ldots\right)$, Corollary 3.2 gives

$$
\begin{aligned}
& (n-3)(n-4) D_{12}^{2} \equiv(-2,(n-4),(n-4), 0, \ldots), \\
& (n-3)(n-4) D_{13}^{2} \equiv(-2,(n-4), 0,(n-4), \ldots), \\
& (n-3)(n-4) D_{23}^{2} \equiv(-2,0,(n-4),(n-4), \ldots),
\end{aligned}
$$

so any effective combination of these has a positive coefficient in at least one of the second or third entries. If $\pi: X_{6+k, 3+k} \rightarrow X_{6,3}$ forgets all the distinguished points other than the first three, and $p: X_{6+k, 3+k} \rightarrow X_{6+k, 3}$ makes indistinguishable all but the first three distinguished points, then

$$
p_{*} \pi^{*} F_{\tau}=(0,0,0, \ldots) .
$$

Thus the effective divisor $p_{*} \pi^{*} F_{\tau}$ is similarly not in the cone generated by boundary divisors of $X_{n, 3}$ for all $n \geq 6$. 
These counterexamples lift to $X_{n, m}$ for any $m \geq 3$ via the (finite) morphisms $X_{n, m} \rightarrow X_{n, 3}$, so

4.2. Proposition. The cone generated by boundary divisors in $N^{1}\left(X_{n, m}\right)$ is a proper subcone of $\overline{N E}^{1}\left(X_{n, m}\right)$ for all $n \geq 6$ and all $m \geq 3$.

5. The Cone of Effective Divisors of $X_{n, 1}$

We will use [Kee92, Theorem 2, p. 561]:

5.1. Theorem (Keel). For any scheme $Y$ the canonical map of Chow rings

$$
A^{*}\left(X_{n}\right) \otimes A^{*}(Y) \rightarrow A^{*}\left(X_{n} \times Y\right)
$$

is an isomorphism.

5.2. Corollary. If $V, W$ are finite images of some $X_{n}, X_{m}$, respectively, then

$$
\begin{aligned}
N^{1}(V \times W) & \cong p_{1}^{*} N^{1}(V) \times p_{2}^{*} N^{1}(W) \\
\text { and so } \quad \overline{N E}^{1}(V \times W) & \cong p_{1}^{*} \overline{N E}^{1}(V) \times p_{2}^{*} \overline{N E}^{1}(W),
\end{aligned}
$$

where $p_{i}$ is the ith projection.

For notational simplicity, we make the following definitions for divisors on $X_{n, m}$ $(0 \leq m \leq 2)$ :

Definitions. (1) Set $B_{k}:=D_{\{1, \ldots, m\}}^{k}(2 \leq k \leq n-2)$. Note that when $m=0$, $B_{n-k}=B_{k}$.

(2) It is straightforward to show that divisorial ramification of the quotient morphism $X_{n} \rightarrow X_{n, m}$ occurs only above $B_{n-2}$, and the ramification is simple (see KM96] for a full discussion). Thus, set $b_{n-2}:=\frac{1}{2} B_{n-2}$, and $b_{i}:=B_{i}$ for all other $i$. It is standard practice in the intersection theory on $\bar{M}_{g}$ to deal with ramification at this stage (cf. $\delta_{1}=\frac{1}{2} \Delta_{1}$ ); using the $b$ 's instead of the $B$ 's accounts for the translation between intersection calculations in $X_{n}$ and $X_{n, m}$. The notation also simplifies formulae by allowing all the boundary classes to be treated in a symmetric way.

(3) For $2 \leq k \leq n-3$ let $C_{k}$ be the curve class obtained by varying the node of a general member of $B_{k}$ along the component not containing any distinguished marked point.

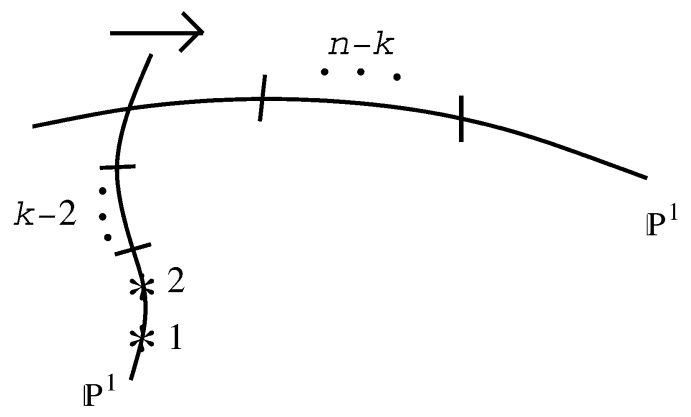

FIgURE 1. The one-parameter family $C_{k}$. 
Notation. Given divisor classes $d_{1}, \ldots, d_{k} \in N^{1}(X)$ and a curve class $C \in N_{1}(X)$,

$$
\text { " } C \equiv \sum n_{i} \check{d}_{i} "
$$

will mean $C . d_{i}=n_{i}$ for each $i$. If the $d_{i}$ form part of a basis of $N^{1}(X), \check{d}_{i}$ will denote the dual to $d_{i}$ in the dual basis, and (3) will further mean that the basis elements whose duals do not appear intersect $C$ trivially.

Now fix $m=1$. The $\left\{b_{i}\right\}$ give a basis for $N^{1}\left(X_{n, 1}\right)$; we formally define $\check{b}_{1}=0$. Define $C_{1}$ to be the curve class of a fibre of the morphism $X_{n, 1} \rightarrow X_{n-1,0}$ forgetting the distinguished marked point. Then

$$
C_{k} \equiv(n-k) \check{b}_{k+1}+(2-n+k) \check{b}_{k} \quad(1 \leq k \leq n-3) .
$$

$C_{k} \in N e f_{1}\left(B_{k}\right)$ for $k \geq 2$, and $C_{1} \in N e f_{1}\left(X_{n, 1}\right)$. Thus any prime divisor $D$ having class not proportional to that of any boundary divisor has $D . C_{k} \geq 0$ for all $k=1, \ldots, n-3$. Rewriting (4) as

$$
\check{b}_{k+1} \equiv \frac{1}{n-k}\left[C_{k}+(n-k-2) \check{b}_{k}\right] \quad(1 \leq k \leq n-3),
$$

we see inductively that the coordinates of any such $D$ are non-negative, so

5.3. Proposition $(n \geq 4) \cdot \overline{N E}^{1}\left(X_{n, 1}\right)$ is simplicial, generated by the boundary classes $B_{i}(i=2, \ldots, n-2)$.

$$
\text { 6. } \operatorname{Nem}\left(X_{n, 0}\right)
$$

We use Proposition 5.3 to calculate $\operatorname{Nem}\left(X_{n, 0}\right)$.

6.1. Lemma. $\overline{N E}^{1}\left(X_{n, 0}\right)$ is generated by its boundary divisors.

Proof. This is shown in [KM96]; it is also an immediate corollary of Proposition 5.3,

The boundary divisors of $X_{n, 0}$ are of the form $X_{l+1,1} \times X_{n-l+1,1}$, so by Lemma 6.1 and Theorem 5.1 we need only consider contributions from $X_{l+1,1}(3 \leq$ $l \leq n-2)$. More generally, we will calculate the contribution of $X_{l+1,1}$ to the inequalities determining $N e m\left(X_{n, m}\right), m=0,1,2$. For $m=0,1$, a basis is given by the set of all $b_{i}$ (note that $b_{n-k}=b_{k}$ when $m=0$ ); we formally define $\check{b}_{1}:=0$. For $m=2$ we will use $b_{3}, \ldots, b_{n-2}$ as part of a basis for $N^{1}\left(X_{n, 2}\right)$, and formally define $\check{b}_{1}=\check{b}_{2}=0$.

For $3 \leq l \leq n-2, l \leq n-m$, and $0 \leq m \leq 2$ let

$$
q: X_{l+1,1} \rightarrow X_{n, m}
$$

be the inclusion given by attaching to the distinguished marked point of every member of $X_{l+1,1}$ a fixed element of $X_{n-l+1, m+1}$ at its highest numbered distinguished point. One checks $(1 \leq k \leq l-1)$ :

$$
q_{*} C_{k} \equiv(l-k+1) \check{b}_{n-l+k}-(l-k-1) \check{b}_{n-l+k-1},
$$

giving the recursive relationship

$$
q_{*} \check{b}_{k+1}=\check{b}_{n-l+k}-\left(\frac{l-k-1}{l-k+1}\right) \check{b}_{n-l+k-1}+\left(\frac{l-k-1}{l-k+1}\right) q m_{l+1 *} \check{b}_{k} .
$$


The recursion telescopes,

$$
q_{*} \check{b}_{k+1}=\check{b}_{n-l+k}-\frac{(l-k-1)(l-k)}{l(l-1)} \check{b}_{n-l}
$$

giving the non-negative half-planes $(1 \leq k \leq l-2,3 \leq l \leq n-2)$

$$
l \cdot(l-1) \check{b}_{n-l+k} \geq(l-k-1)(l-k) \check{b}_{n-l} .
$$

The inequalities for $k=1$ generate, so (recalling for $m=2$ that $\check{b}_{2}:=0$ )

6.2. Proposition $(m=0,1,2)$. The contribution from the spaces $X_{l+1,1}$ to the inequalities determining $\mathrm{Nem}\left(X_{n, m}\right)$ are

$$
l \cdot \check{b}_{n-l+1} \geq(l-2) \cdot \check{b}_{n-l} \quad(3 \leq l \leq n-2) .
$$

For $m=0, b_{n-k}=b_{k}$, and Proposition 6.2 and Lemma 6.1 imply:

6.3. Theorem $(n \geq 6)$. Nem $\left(X_{n, 0}\right)$ is determined by the $2\left(\left\lfloor\frac{n}{2}\right\rfloor-2\right)$ inequalities $\left(2 \leq i \leq\left\lfloor\frac{n}{2}\right\rfloor-1\right)$ :

$$
\left(\frac{n-i-2}{n-i}\right) \check{b}_{i} \leq \check{b}_{i+1} \leq\left(\frac{i+1}{i-1}\right) \check{b}_{i} .
$$

In particular, any moving linear system of $X_{n, 0}$ is big.

Because of the simplicity of these inequalities, we can explicitly describe the cone $\operatorname{Nem}\left(X_{n, 0}\right)$.

6.4. Corollary. $N e m\left(X_{n, 0}\right)$ is generated by $2^{\left\lfloor\frac{n}{2}\right\rfloor-2}$ rays, which are inductively described in terms of the basis $\left\{\breve{b}_{i}\right\}$ as follows. Let the first entry of a generating vector be 1 . Then given that the ith entry is a, there are two choices for the $i+1$ st, namely

$$
a \cdot\left(\frac{n-i-2}{n-i}\right) \quad \text { and } \quad a \cdot\left(\frac{i+1}{i-1}\right) .
$$

Remark. For any $i \in\left\{2, \ldots,\left\lfloor\frac{n}{2}\right\rfloor\right\}$, there is an extremal ray $R_{i} \subseteq N e m\left(X_{n, 0}\right)$ which, when restricted to each of the $B_{j}$, is big only on $B_{i}$. One constructs a vector $v_{i}$ generating $R_{i}$ as follows: let the left-hand inequalities of Theorem 6.3 be called "Type A" and the others "Type B." Set the ith entry of $v_{i}$ equal to 1 , and use the inequalities of Type A (as equalities) to determine the entries to the right of the $i t h$, and those of Type B to determine those to the left.

The $R_{i}$ are interesting from the point of view of Mori theory: if they were generated by moving linear systems having finitely generated section rings (in particular if the $X_{n, 0}$ were Mori Dream Spaces), they would correspond to birational maps $\varphi_{i}: X \rightarrow Z_{i}$ contracting all the $B_{j}$ except $B_{i}$; the targets $Z_{i}$ are $\mathbb{Q}$-factorial of Picard number one (see [HK00] for background on section rings and "contracting" rational maps).

6.5. Examples. We illustrate cross-sections of $\overline{N E}^{1}\left(X_{n, 0}\right)$ for $6 \leq n \leq 9$. In the following, we use as ordered bases $\left(b_{2}, \ldots, b_{\left\lfloor\frac{n}{2}\right\rfloor}\right)$. For comparison purposes, we have included the calculations of $\mathrm{Nef}^{1}\left(X_{n, 0}\right)$ done in [KM96]. Moving and nem cones are the same for $n \leq 6$, but this is not known for $n \geq 7$.

$\mathbf{n}=\mathbf{6}: \operatorname{Nem}\left(X_{6,0}\right)=\operatorname{Nef}^{1}\left(X_{6,0}\right)$ is generated by $(2,1)$ and $(1,3)$.

$\mathbf{n}=\mathbf{7}: \operatorname{Nem}\left(X_{7,0}\right)$ is generated by $(5,3)$ and $(1,3) . N^{1} f^{1}\left(X_{7,0}\right)$ is generated by $(1,3)$ and $(1,1)$. 


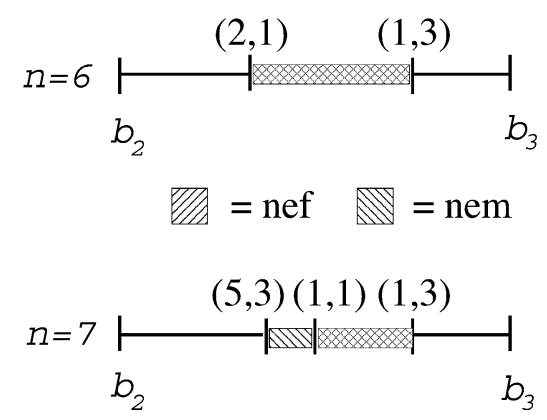

Figure 2. Cross sections of $\overline{N E}^{1}\left(X_{6,0}\right)$ and $\overline{N E}^{1}\left(X_{7,0}\right)$.

$\mathbf{n}=\mathbf{8}: \operatorname{Nem}\left(X_{8,0}\right)$ is generated by

$A:=(3,2,4), \quad C:=(1,3,6), \quad Q:=(5,15,9), \quad R:=(15,10,6)$.

$N e f^{1}\left(X_{8,0}\right)$ is spanned by $A$ and $C$ together with $P:=(6,11,8)$ and $B:=$ $(2,6,5)$.

Remark. The three vertices $A, B$, and $C$ come from the nef cone of $\bar{M}_{3}$ under the natural identification of $X_{8}$ with the hyperelliptic locus in $\bar{M}_{3}$ Rul01.

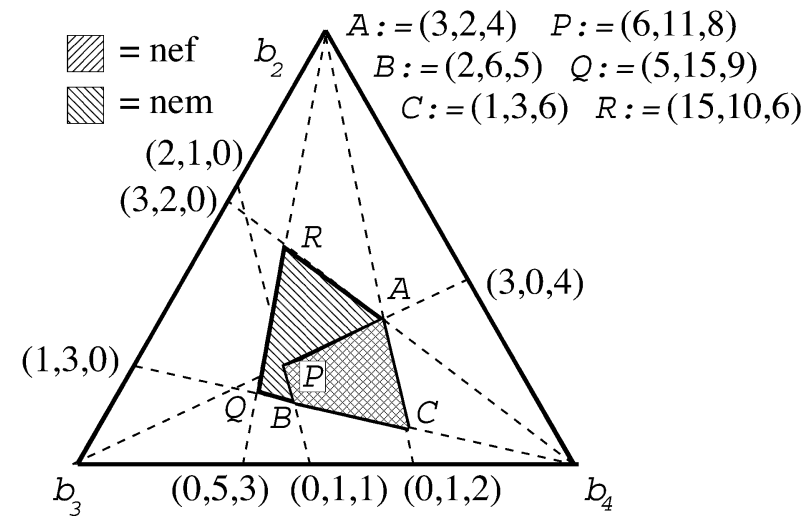

Figure 3. A cross section of $\overline{N E}^{1}\left(X_{8,0}\right)$.

$\mathbf{n}=\mathbf{9}: \operatorname{Nem}\left(X_{9,0}\right)$ is generated by

$B:=(1,3,2), \quad C:=(1,3,6), \quad T:=(7,5,10), \quad U:=(21,15,10)$.

$N e f^{1}\left(X_{9,0}\right)$ is spanned by $B$ and $C$ together with $A:=(1,1,2)$ and $S:=$ $(3,3,4)$.

7. The Cone of EFfeCtive Divisors of $X_{n, 2}$

For notational ease, define $b_{i}^{*}:=D_{\{1\}}^{i}$ in $X_{n, 2}$. From Corollary 3.2 a basis for $N^{1}\left(X_{n, 2}\right)$ is given by the set of all $\left\{b_{i}, b_{i}^{*}\right\}$ excluding $b_{2}$. Recall that in $N_{1}\left(X_{n, 2}\right)$ we formally define $\check{b}_{1}=\check{b}_{2}=0$; we also set $\check{b}_{1}^{*}=0$. 


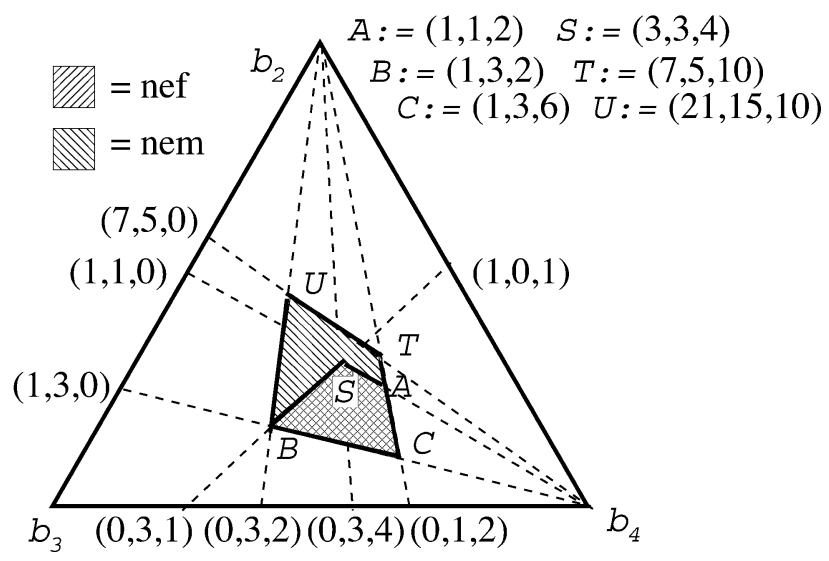

Figure 4. A cross section of $\overline{N E}^{1}\left(X_{9,0}\right)$.

Let $D$ be any prime divisor having class not proportional to that of any boundary. The boundaries of $X_{n, 2}$ are of the form $X_{l+1,3} \times X_{n-l+1,1}$ or $X_{l+1,2} \times X_{n-l+1,2}$. Thus the restriction to $D$ to any appropriate factor $X_{l+1, m}(1 \leq m \leq 3)$ must be effective. Constraints coming from spaces of the form $X_{l+1,1}$ were determined in Proposition 6.2, in particular, $\check{b}_{i} . D \geq 0(3 \leq i \leq n-2)$.

Next consider constraints coming from the spaces $X_{l+1,2}(2 \leq l \leq n-2)$.

7.1. Lemma. The subcone of $N^{1}\left(X_{l+1,2}\right)$ generated by the classes $\left\{b_{i}, b_{i}^{*}\right\}_{i=2}^{l-1}$ is determined by the inequalities $(2 \leq j \leq l-1,3 \leq i \leq l-1)$ :

$$
\begin{gathered}
\check{b}_{j}^{*} \geq 0 \quad \text { and } \\
(j-1)(l-j) \check{b}_{i}+(l-i+1)(l-i) \check{b}_{j}^{*} \geq 0 .
\end{gathered}
$$

Proof. This follows from Corollary 3.2 .

Definition. Define the curve class $C_{1}^{*}$ by varying the marked point 1 along a general element of $X_{l+1,2}$. For $2 \leq i \leq l-2$ define $C_{i}^{*}$ by varying the component of a general element of $b_{i}^{*} \subseteq X_{l+1,2}$ with the marked point 1 and $i-1$ indistinguishable marked points along the component with the remaining marked point.

Thus, $C_{i}^{*} \equiv \check{b}_{i+1}+(l-i) \check{b}_{i+1}^{*}+(1-l+i) \check{b}_{i}^{*}$, or

$$
\check{b}_{i+1}^{*} \equiv \frac{1}{l-i}\left(C_{i}^{*}-\check{b}_{i+1}+(l-i-1) \check{b}_{i}^{*}\right) \quad(1 \leq i \leq l-2) .
$$

Consider the morphism $r: X_{l+1,2} \rightarrow X_{n, 2}$ which attaches a fixed element $\left(C, \bar{P}_{1}, \bar{P}_{2}\right) \in X_{n-l+1,2}$ to each element $\left(C, P_{1}, P_{2}\right) \in X_{l+1,2}$ by identifying $P_{2}$ with $\bar{P}_{1}$. Then a calculation similar to that done in $\$ 6$ shows

$$
r_{*} \check{b}_{i+1}^{*} \equiv \check{b}_{i+1}^{*}+\frac{i(l-i-1)}{(l-2)(l-1)} \check{b}_{n-l+1}-\frac{i}{l-1} \check{b}_{l}^{*} \quad(1 \leq i \leq l-2) .
$$

Pushing forward the inequalities $\check{b}_{j}^{*} \geq 0$ of Lemma 7.1 gives

$$
\check{b}_{j}^{*}+\frac{(j-1)(l-j)}{(l-2)(l-1)} \check{b}_{n-l+1} \geq\left(\frac{j-1}{l-1}\right) \check{b}_{l}^{*} \quad(2 \leq j \leq l-1) .
$$


Fixing $l=n-2$ we get necessary inequalities for the prime divisor $D$ :

$$
(n-4)(n-3) \check{b}_{j}^{*}+(j-1)(n-j-2) \check{b}_{3} \geq(n-4)(j-1) \check{b}_{n-2}^{*} .
$$

If we reverse the roles of the two marked points (in the definition of $C_{i}^{*}$ and the morphism $r_{k}$ ) we get

$$
(n-4)(n-3) \check{b}_{n-j}^{*}+(j-1)(n-j-2) \check{b}_{3} \geq(n-4)(j-1) \check{b}_{2}^{*} .
$$

Replacing $j$ with $n-j$ in (9) gives

$$
(n-4)(n-3) \check{b}_{j}^{*}+(n-j-1)(j-2) \check{b}_{3} \geq(n-4)(n-j-1) \check{b}_{2}^{*} .
$$

Note that (8)-(10) continue to hold for $j=n-2$, so apply for $2 \leq j \leq n-2$.

Varying the point of attachment of the component with $n-3$ undistinguished marked points of a general element of $B_{3} \subseteq X_{n, m}$ along the other component gives

$$
\check{b}_{2}^{*}+\check{b}_{n-2}^{*} \geq \check{b}_{3},
$$

satisfied by any prime $D$ as above. Then $\breve{b}_{j}^{*} \geq 0(2 \leq j \leq n-2)$ comes from $(n-j-1)[$ [neq. (8) $]+(j-1)[$ Ineq. (10)] $]+(n-j-1)(j-1)(n-4)$ [Ineq. (11)].

7.2. Proposition $(n \geq 4) \cdot \overline{N E}^{1}\left(X_{n, 2}\right)$ is generated by boundary divisors. Further, $\operatorname{Nem}\left(X_{n, 2}\right)$ is contained in the (simplicial) cone generated by the classes $\left\{b_{i}, b_{j}^{*}\right\}$ excluding $b_{2}$.

7.3. Example. Consider the case $n=5$. From Corollary $3.2 \rho\left(X_{5,2}\right)=3$ and

$$
3 b_{2} \equiv b_{2}^{*}+b_{3}^{*}-b_{3}
$$

$\overline{N E}^{1}\left(X_{5,2}\right)$ is generated by the four boundaries $b_{2}, b_{3}, b_{2}^{*}$, and $b_{3}^{*} . N e f^{1}\left(X_{5,2}\right)$ is the dual of this cone; since $X_{5,2}$ is a surface, its nem, moving, and nef cones are identified (Figure 5).

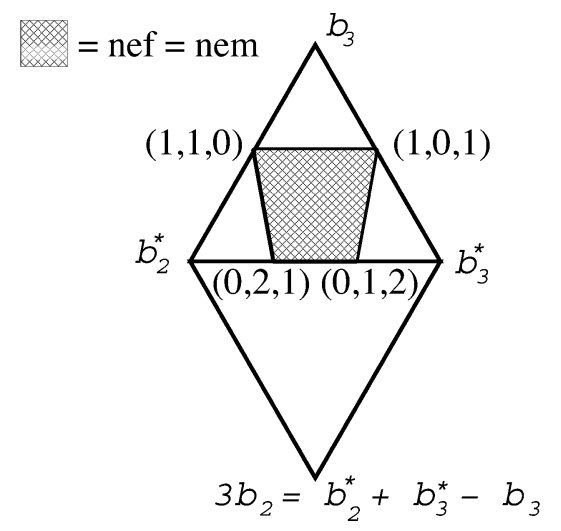

Figure 5. A cross section of $\overline{N E}^{1}\left(X_{5,2}\right)$. 


\section{8. $\operatorname{Nem}\left(X_{n, 1}\right)$}

We use Proposition 7.2 to calculate $\operatorname{Nem}\left(X_{n, 1}\right)$. The boundaries of $X_{n, 1}$ are of the form $X_{l+1,2} \times X_{n-l+1,1}$. Proposition 6.2 gives the contributions from the $X_{l+1,1}$ :

$$
l \cdot \breve{b}_{n-l+1} \geq(l-2) \cdot \check{b}_{n-l} \quad(3 \leq l \leq n-2) .
$$

If $s: X_{l+1,2} \rightarrow X_{n, 1}$ is a corresponding attaching map, we adapt (6) and (7) to give $(2 \leq i \leq l-2)$ :

$$
\begin{aligned}
s_{*} \check{b}_{i+1} & \equiv \check{b}_{n-l+i}-\frac{(l-i-1)(l-i)}{(l-2)(l-1)} \check{b}_{n-l+1} \\
\text { and } \quad s_{*} \check{b}_{i+1}^{*} & \equiv \check{b}_{i+1}+\frac{i(l-i-1)}{(l-2)(l-1)} \check{b}_{n-l+1}-\frac{i}{l-1} \check{b}_{l} .
\end{aligned}
$$

Pushing forward the inequalities determining $\overline{N E}^{1}\left(X_{l+1,2}\right)$ (Lemma 7.1 and Proposition (7.2) gives

$$
\begin{aligned}
(l-1)(j-1)(l-j) \check{b}_{n-l+i-1}+(l-1)(l-i)(l-i+1) \check{b}_{j} & \\
& \geq(j-1)(l-i)(l-i+1) \check{b}_{l} \quad(2 \leq i, j \leq l-1) .
\end{aligned}
$$

8.1. Corollary. $\operatorname{Nem}\left(X_{n, 1}\right)$ is generated by the $(n-1)(n-4) / 2$ inequalities $(3 \leq$ $l \leq n-2,2 \leq j \leq l-1)$ :

$$
\begin{gathered}
l \cdot \check{b}_{n-l+1} \geq(l-2) \check{b}_{n-l} \quad \text { and } \\
(j-1)(l-j) \check{b}_{n-l+1}+(l-1)(l-2) \check{b}_{j} \geq(j-1)(l-2) \check{b}_{l} .
\end{gathered}
$$

Proof. $\operatorname{Nem}\left(X_{n, 1}\right)$ is by definition a subset of $\overline{N E}^{1}\left(X_{n, 1}\right)$, so the inequalities $\breve{b}_{i} \geq 0$ must be included in its calculation. These however are subsumed by (12) and (14): let $I_{i, j, l}$ refer to the inequality (14). If $n$ is odd, " $\breve{b}_{2} \geq 0$ " comes from $I_{2,2,(n+1) / 2}$; if $n$ is even, " $\check{b}_{2} \geq 0$ " comes from $\left(\frac{n}{2}\right) I_{2,2, n / 2}+\left(\frac{n}{2}-2\right) I_{2,2, n / 2+1}$. The other inequalities " $\check{b}_{i} \geq 0$ " then follow from (12).

Denote the inequalities (12) by $J_{l}$. For $i \geq 3$,

$$
I_{i, j, l}=a J_{l-i+2}+b I_{i-1, j, l}
$$

where

$$
a=\frac{(l-1)(j-1)(l-j)}{(l-i+2)} \quad \text { and } \quad b=\frac{l-i}{l-i+2} .
$$

Thus we need only the $J_{l}$ and the $I_{2, j, l}$.

We now study some properties of $\operatorname{Nem}\left(X_{n, 1}\right)$. From the inequalities (12) note that if an element of $\operatorname{Nem}\left(X_{n, 1}\right)$ has $\check{b}_{i}=0$, then it also has $\check{b}_{j}=0$ for all $j<i$. Suppose that $\check{b}_{3}=0$. Then $I_{2,2, n-2}$ implies $0 \geq \check{b}_{n-2}$, so that all $\check{b}_{i}=0$. Thus the only possible zero coordinate of a vector $(v \neq 0) \in \operatorname{Nem}\left(X_{n, 1}\right)$ is the first: $\check{b}_{2}(v)=0$. This implies $v$ trivially intersects a fibre of $\pi: X_{n, 1} \rightarrow X_{n-1,0}$, so we expect $v$ to be pulled back from $\operatorname{Nem}\left(X_{n-1,0}\right)$. In fact, if $\check{b}_{2}=0$, then $I_{2,2, l}$ implies

$$
\check{b}_{n-l+1}=\check{b}_{l} \quad(3 \leq l \leq n-2) ;
$$

together with the inequalities $J_{l}$, this forces

$$
\frac{n-l-2}{n-l} \check{b}_{l} \leq \check{b}_{l+1} \leq \frac{l}{l-2} \check{b}_{l} .
$$


Since $\pi^{*} b_{l}=b_{l+1}+b_{n-l+1}\left(\right.$ except $\pi^{*} b_{\frac{n}{2}}=b_{\frac{n}{2}+1}$ for $n$ even) we get from Theorem 6.3 8.2. Proposition. $\operatorname{Nem}\left(X_{n, 1}\right)=\pi^{*} \operatorname{Nem}\left(X_{n-1,0}\right)+C$, where every element of $C$ is big.

Thus any fibration of any space $V$ isomorphic to $X_{n, 1}$ in codimension one contracts the (images of the) fibres of $\pi: X_{n, 1} \rightarrow X_{n-1,0}$.

8.3. Examples. We illustrate cross sections of $\overline{N E}^{1}\left(X_{n, 1}\right)$ for $5 \leq n \leq 7$. In the following we again include the computations of nef cones from [KM96].

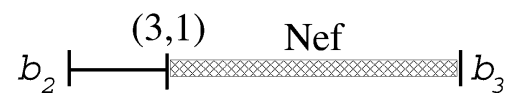

Figure 6. A cross section of $\overline{N E}^{1}\left(X_{5,1}\right)$.

$\mathbf{n}=\mathbf{5}$ : From Corollary 8.1, $\operatorname{Nem}\left(X_{5,1}\right)$ is determined by $3 \check{b}_{3} \geq \check{b}_{2}$ and $\check{b}_{2} \geq 0$. Since $X_{5,1}$ is a surface, this also gives the nef cone (Figure 6 ). $\mathbf{n}=\mathbf{6}$ : From Corollary 8.1, $N e m\left(X_{6,1}\right)$ is determined by

$$
\begin{array}{lrl}
3 \check{b}_{4} & \geq \check{b}_{3}, & 2 \check{b}_{4}+4 \check{b}_{2} \geq 2 \check{b}_{3}, \\
4 \check{b}_{3} \geq 2 \check{b}_{2}, & 6 \check{b}_{3}+18 \check{b}_{2} \geq 6 \check{b}_{4},
\end{array}
$$

These determine the cone spanned by the vectors

$$
(6,3,1), \quad(1,3,1), \quad(0,1,1), \quad(1,3,6), \quad(2,1,2) .
$$

The nef cone is given by the span of

$$
(6,3,1), \quad(1,3,1), \quad(0,1,1), \quad(2,1,2) .
$$

Remark. $(6,3,1)$ corresponds to (i.e. is a multiple of the pull-back of) the relative dualizing sheaf $\omega$ of $\bar{M}_{2,1}$ under the identification of $X_{6,1}$ with the Weierstrass locus of $\bar{M}_{2,1}$ (see 10 for more information), $(2,1,2)$ corresponds to the determinant of the Hodge bundle $\lambda$, and $(1,3,1)$ corresponds to $12 \lambda-\delta_{i r r}$, where $\delta_{i r r}$ denotes the class of the closure of the locus of irreducible elements of $\bar{M}_{2,1}$ with a single node.

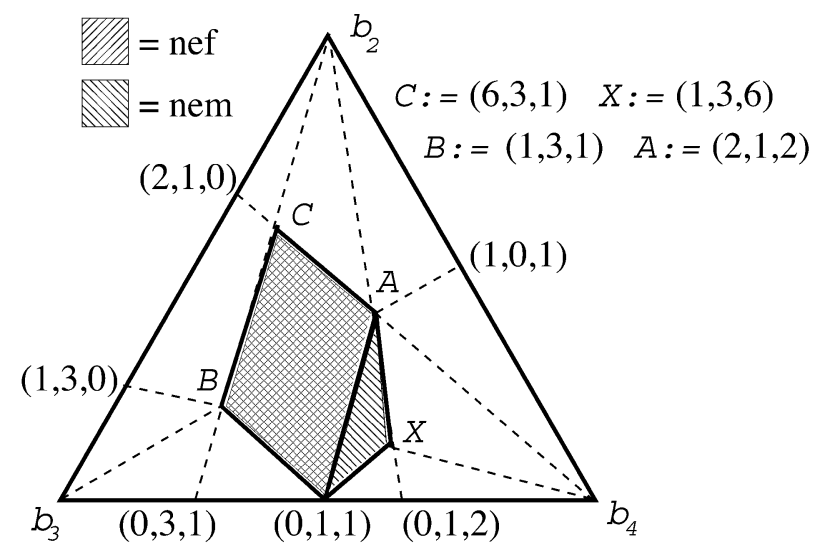

Figure 7. A cross section of $\overline{N E}^{1}\left(X_{6,1}\right)$. 
$\mathbf{n}=\mathbf{7}$ : From Corollary 8.1, $\operatorname{Nem}\left(X_{7,1}\right)$ is determined by

$$
\begin{aligned}
& 3 \check{b}_{5} \geq \check{b}_{4}, \quad 5 \check{b}_{3} \geq 3 \check{b}_{2}, \quad \check{b}_{2} \geq 0, \quad 8 \check{b}_{3} \geq 3 \check{b}_{5}, \\
& 4 \check{b}_{4} \geq 2 \check{b}_{3}, \quad 2 \check{b}_{5}+4 \check{b}_{2} \geq 2 \check{b}_{3}, \quad \check{b}_{3}+4 \check{b}_{2} \geq \check{b}_{5}, \quad \check{b}_{3}+4 \check{b}_{4} \geq 3 \check{b}_{5} \text {. }
\end{aligned}
$$

This gives $\mathrm{Nem}\left(X_{7,1}\right)$ as the convex hull of

$$
\begin{array}{llrrr}
(5,12,36,32), & (10,6,3,6), & (5,3,9,8), & (5,12,6,2), & (0,1,3,1), \\
(5,12,21,32), & (10,6,3,1), & (5,3,9,3), & (20,12,21,32), & (0,2,1,2) .
\end{array}
$$

$N e f^{1}\left(X_{7,1}\right)$ is the cone over

$$
(10,6,3,1), \quad(5,3,4,3), \quad(5,12,6,2), \quad(0,1,3,1), \quad(0,2,1,2) .
$$

Remarks. (a) $(0,2,1,2)$ corresponds to $\lambda$ under the morphism $p: X_{7,1} \rightarrow \bar{M}_{2,1}$ described in 10 . Also, $(0,1,3,1)$ corresponds to $12 \lambda-\delta_{i r r}$, and $(5,12,6,2)$ corresponds to $\omega$.

(b) It is easy to check that the ray generated by $(10,6,3,1)$ is the push-forward under $\pi_{7}: X_{7} \rightarrow X_{7,1}$ of the ray generated by

$$
\begin{aligned}
L_{7} \equiv D_{12}+D_{37}+D_{47}+D_{57}+D_{67}+D_{347}+D_{357}+ \\
D_{367}+D_{457}+D_{467}+D_{567}+D_{3457}+D_{3467}+D_{3567}+D_{4567},
\end{aligned}
$$

which corresponds to a hyperplane section from $\mathbb{P}^{4}$ under the blow-up description $f: \bar{M}_{0,7} \rightarrow \mathbb{P}^{4}$ in [HT02. $\left|L_{7}\right|$ contains elements missing any given fibre of $\pi_{7}$, so $(10,6,3,1)$ moves in $X_{7,1}$. We use this in the proof of Proposition 10.2

\section{Relations with $\bar{M}_{g}$ and $\bar{M}_{g, 1}$}

We use the notation of [Fab99] for the standard divisor classes on $\bar{M}_{g}$ and $\bar{M}_{g, 1}$, except on $\bar{M}_{g, 1}$ we abbreviate $\delta_{i ;\{1\}}$ by $\delta_{i}(1 \leq i \leq g-1)$, and $\omega_{\pi_{1}}$ by $\omega$. It is a fact that the classes $\delta_{i r r}, \delta_{1}, \ldots, \delta_{\left\lfloor\frac{g}{2}\right\rfloor}$ and $\lambda$ generate $N^{1}\left(\bar{M}_{g}\right)$, and the classes $\delta_{i r r}, \delta_{1}$, $\ldots, \delta_{g-1}, \lambda$, and $\omega$ generate $N^{1}\left(\bar{M}_{g, 1}\right)$ in any characteristic [DM69, [Mor01. These classes are independent for $g \geq 3$.

To avoid pathologies with hyperelliptic curves, we restrict to characteristic $c \neq 2$ in this and the following section.

\subsection{The hyperelliptic locus of $\bar{M}_{g}$. Consider the natural morphism}

$$
i: X_{2 g+2,0} \rightarrow \bar{M}_{g}
$$

identifying the source with the hyperelliptic locus of $\bar{M}_{g}$, by associating to each element of $X_{2 g+2,0}$ its (unique) degree-two admissible cover [HM98, pp. $180 \mathrm{ff}$.]. For $n \geq 3$ we use the ordered basis $\left(\lambda, \delta_{i r r}, \delta_{1}, \ldots, \delta_{\left\lfloor\frac{g}{2}\right\rfloor}\right)$ for $N^{1}\left(\bar{M}_{g}\right)$. The following calculations still hold for $g=2$, but $\check{\lambda}$ should be replaced by $\frac{1}{10} \check{\delta}_{i r r}+\frac{1}{5} \check{\delta}_{1}$ and eliminated as a basis element.

Let $C_{k} \in \overline{N E}_{1}\left(X_{n, 0}\right)$ be as described in $§ 5$ (define $C_{1} \in \overline{N E}_{1}\left(X_{n, 0}\right)$ as the image of $\left.C_{1} \in \overline{N E}_{1}\left(X_{n, 1}\right)\right)$. Defining $\check{\delta}_{0}:=0 \in N^{1}\left(\bar{M}_{g}\right)$, the intersection numbers with $i_{*} C_{2 j+1}$ are

$$
\delta_{i r r}=2(2 g+1-2 j), \quad \delta_{j}=\frac{1}{2}(2 j+1-2 g), \quad \kappa+\delta_{j}=2(g-j-1) .
$$


Using the relation $12 \lambda \equiv \kappa+\delta$ gives

$$
i_{*} C_{2 j+1} \equiv 2(2 g+1-2 j) \check{\delta}_{i r r}+\frac{1}{2}(2 j+1-2 g) \check{\delta}_{j}+\frac{1}{2}(g-j) \check{\lambda} \quad(j \geq 0) .
$$

Similarly,

$$
i_{*} C_{2 j} \equiv 4(j-g) \check{\delta}_{i r r}+(g+1-j) \check{\delta}_{j} \quad(j \geq 1)
$$

By induction,

$$
\begin{aligned}
i_{*} \check{b}_{2 j} & \equiv 2 \check{\delta}_{i r r}+\frac{j(g+1-j)}{4 g+2} \check{\lambda} \quad(1 \leq j \leq g), \\
i_{*} \check{b}_{2 j+1} & \equiv \frac{1}{2} \check{\delta}_{j}+\frac{j(g-j)}{4 g+2} \check{\lambda} \quad(1 \leq j \leq g-1),
\end{aligned}
$$

where $\delta_{j}:=\delta_{g-j}$ for $j>\left\lfloor\frac{g}{2}\right\rfloor$. (These can also be deduced using the formulae given in [HM98, p. 303], but note the ramification of $X_{n} \rightarrow X_{n, 0}$ induced by $\mathcal{S}_{2 g+2}$ is ignored in that calculation; the most economical way to account for the ramification is to replace their $\Xi_{1}$ with $\xi_{1}:=\frac{1}{2} \Xi_{1}$.)

9.1. Corollary $(g \geq 2)$. An effective divisor in $\bar{M}_{g}$ pulls back to $N e m\left(X_{2 g+2,0}\right)$ iff it is contained in the cone generated by the following inequalities $(1 \leq i \leq g-1)$ :

$$
\begin{aligned}
(2 i-1) \check{\delta}_{i} & \leq 4(2 i+1) \check{\delta}_{i r r}+i \check{\lambda}, \\
4 i \check{\delta}_{i r r} & \leq(i+1) \check{\delta}_{i} .
\end{aligned}
$$

Proof. This follows from (16) and Theorem 6.3.

9.2. The morphisms $X_{2 n+3,1} \rightarrow \bar{M}_{g}, \bar{M}_{g, 1}(1 \leq n \leq g-1, g)$. To each element $C \in X_{2 n+3,1}$ there is a unique degree two admissible cover $\widetilde{C}$ obtained as in $\$ 9.1$ via ignoring the distinguished point of $C$. This distinguished point then determines (up to hyperelliptic involution of some component of $\widetilde{C}$ ) a marked point $P \in \widetilde{C}$. Consider the morphism $p: X_{2 n+3,1} \rightarrow \bar{M}_{g}(1 \leq n \leq g-1)$ given by attaching to $\widetilde{C}$ a fixed curve $D$ of complementary genus, by identifying a fixed point $Q \in D$ with $P$. Via a calculation analogous to the one of the previous subsection,

$$
\begin{aligned}
p_{*} C_{1} & \equiv-(n-1) \check{\delta}_{g-n}, \\
p_{*} C_{2 j+1} & \equiv-4(n-j) \check{\delta}_{i r r}+(n+1-j) \check{\delta}_{g-n+j}, \quad \text { and } \\
p_{*} C_{2 j} & \equiv 2(2 n+3-2 j) \check{\delta}_{i r r}-\frac{1}{2}(2 n+1-2 j) \check{\delta}_{g-n+j-1}+\frac{1}{2}(n+1-j) \check{\lambda} .
\end{aligned}
$$

Remark. As the above equations are written, they also apply when the target space is $\bar{M}_{g, 1}$; the morphism $p$ in that case is given by taking some fixed point $(R \neq Q) \in D$ as marked point.

Induction shows:

$$
\begin{aligned}
& p_{*} \check{b}_{2 j+2} \equiv \frac{1}{2} \check{\delta}_{g-n+j}+\frac{j(n-j)}{2(2 n+1)} \check{\lambda}-\frac{(n-j)(2 n+1-2 j)}{(2 n+1)(n+1)} \check{\delta}_{g-n}, \\
& p_{*} \check{b}_{2 j+1} \equiv 2 \check{\delta}_{i r r}+\frac{j(n+1-j)}{2(2 n+1)} \check{\lambda}-\frac{(2 n+1-2 j)(n+1-j)}{(2 n+1)(n+1)} \check{\delta}_{g-n} .
\end{aligned}
$$

These equations also apply for $n=g$, if $\check{\delta}_{0}:=-\check{\omega} \in N^{1}\left(\bar{M}_{g, 1}\right)$. 
9.2. Corollary $(g \geq 3$, and $2 \leq n \leq g-1)$. The subcone of effective divisors of $\bar{M}_{g}$ pulling back to $\operatorname{Nem}\left(X_{2 n+3,1}\right)$ is the subset determined by $(1 \leq k \leq n-1$ and $0 \leq m \leq k-1$, unless specified otherwise):

$$
\begin{gathered}
\bullet(k+1) \check{\delta}_{g-k} \geq 4 k \check{\delta}_{i r r}, \\
\bullet 4(2 k+1) \check{\delta}_{i r r}+k \check{\lambda} \geq(2 k-1) \check{\delta}_{g-k}, \\
\bullet(2 m+1)(k-m) \check{\delta}_{g-k}+k m(k-m) \check{\lambda}^{2}+k(2 k+1) \check{\delta}_{g-n+m} \\
\geq k(2 m+1) \check{\delta}_{g-n+k}+4 k(k-m) \check{\delta}_{g-n}, \\
\bullet m(k+1)(k-m) \check{\lambda}+(k+1)(2 k+1) \check{\delta}_{g-n+m} \geq 4 m(2 m+1) \check{\delta}_{i r r} \quad(0 \leq m \leq k), \\
+(2 k+1)[2(k-m)+1] \check{\delta}_{g-n} \quad(k) \quad(k-m) \check{\lambda}^{2}+4 k(2 k+1) \check{\delta}_{i r r} \\
\bullet(m+1)[2(k-m)-1] \check{\delta}_{g-k}+k(m+1)(k-1) \check{\delta}_{g-n}+2 k(m+1) \check{\delta}_{g-n+k} .
\end{gathered}
$$

Remark. The corollary also applies (with the same notation) when the target space is $\bar{M}_{g, 1}$. In that case, $g \geq 2,2 \leq n \leq g$, and $\check{\delta}_{0}:=-\check{\omega}$. For $g=2, \check{\lambda}$ should be replaced by $\frac{1}{10} \check{\delta}_{i r r}+\frac{1}{5} \check{\delta}_{1}$.

Proof. Pushing forward the inequalities defining $N e m\left(X_{2 n+3,1}\right)$ (Corollary 8.1) shows the subcone in question is determined by $(1 \leq k \leq n-1$ and $0 \leq m \leq k-1$, unless specified otherwise):

$$
\begin{gathered}
(\mathbf{i})(k+1) \check{\delta}_{g-k} \geq 4 k \check{\delta}_{i r r}, \\
(\mathbf{i i}) 4(2 k+1) \check{\delta}_{i r r}+k \check{\lambda} \geq(2 k-1) \check{\delta}_{g-k} \quad(1 \leq k \leq n), \\
(\mathbf{I})(2 m+1)(k-m) \check{\delta}_{g-k}+k m(k-m) \check{\lambda}+k(2 k+1) \check{\delta}_{g-n+m} \\
\geq k(2 m+1) \check{\delta}_{g-n+k}+4 k(k-m) \check{\delta}_{g-n}, \\
(\mathbf{I I}) 4[2(k+m)+3] \check{\delta}_{i r r}+(k+1)(m+1) \check{\lambda} \geq 2(2 k+1) \check{\delta}_{g-n}, \\
(\mathbf{I I I}) m(k+1)(k-m) \check{\lambda}+(k+1)(2 k+1) \check{\delta}_{g-n+m} \geq 4 m(2 m+1) \check{\delta}_{i r r} \\
+(2 k+1)[2(k-m)+1] \check{\delta}_{g-n} \quad(0 \leq m \leq k, 0 \leq k \leq n-1), \\
(\mathbf{I V})(m+1)[2(k-m)-1] \check{\delta}_{g-k}+k(m+1)(k-m) \check{\lambda}+4 k(2 k+1) \check{\delta}_{i r r} \\
\geq 2 k[2(k-m)-1] \check{\delta}_{g-n}+2 k(m+1) \check{\delta}_{g-n+k} .
\end{gathered}
$$

Fix a positive integer $n \geq 2$. For $n=2$ set $c_{1}:=1$ and $c_{2}:=2$. For $n \geq 3$ set

$$
\begin{aligned}
& c_{1}:=2 n(n-1)[2(k+m)+3]-2(4 n-3)(k+1)(m+1), \\
& c_{2}:=10(k+1)(m+1)-4[2(k+m)+3] .
\end{aligned}
$$

Then the $c_{i}$ are non-negative for $1 \leq k \leq n-1,0 \leq m \leq k-1$, and (denoting e.g. inequality (i) evaluated at $k=1$ by $(\mathbf{i})(1))$

$$
c_{1}[(\mathbf{i})(1)+2(\mathbf{i i})(1)]+c_{2}[(2 n-3)(\mathbf{i})(n-1)+n(\mathbf{i i})(n-1)]
$$

gives

$$
\begin{gathered}
2\left(5 n^{2}-13 n+6\right) \cdot\left[4[2(k+m)+3] \check{\delta}_{i r r}+(k+1)(m+1) \check{\lambda}\right] \geq 0 . \\
5 n^{2}-13 n+6>0 \text { for } n \geq 3, \text { so }(3 \leq n, 1 \leq k \leq n-1,0 \leq m \leq k-1): \\
4[2(k+m)+3] \check{\delta}_{i r r}+(k+1)(m+1) \check{\lambda} \geq 0 .
\end{gathered}
$$


Note that " $\check{\delta}_{g-n} \leq 0$ " comes from $\mathbf{I I I}(1,0)$, so (II), III $(0,0)$, and (ii) $(n)$ are subsumed.

\section{The cone of Effective Divisors of $\bar{M}_{2,1}$}

As an example we analyze $\overline{N E}^{1}\left(\bar{M}_{2,1}\right)$ in terms of $\overline{N E}^{1}\left(X_{7,1}\right)$. Let

$$
p: X_{7,1} \rightarrow \bar{M}_{2,1}
$$

be the morphism described in the first Remark of 99.2 , for $n=g=2 ; p$ is easily seen to be birational. We continue to restrict to characteristic $\neq 2$ in this section.

Let $W \subseteq \bar{M}_{2,1}$ denote the Weierstrass divisor, i.e. the closure of the locus $\{(C, P): P \in C$ is a Weierstrass point $\}$. The images of the boundary divisors of $X_{7,1}$ are

$$
\begin{array}{lll}
B_{2} \mapsto W, & & B_{4} \mapsto \Delta_{1 ;\{1\}}, \\
B_{3} \mapsto\{\text { banana curves }\}, & & B_{5} \mapsto \Delta_{i r r} .
\end{array}
$$

The general element of the locus of "banana curves" is a genus one curve attached at two points to a smooth rational curve with marked point.

From Proposition 5.3, $\overline{N E}^{1}\left(X_{7,1}\right)=\sum_{i=2}^{5} \mathbb{R}^{\geq 0} B_{i}$.

10.1. Corollary. $\overline{N E}^{1}\left(\bar{M}_{2,1}\right)$ is simplicial, generated by the classes of $\Delta_{i r r}, \Delta_{1}$ and $W$.

Proof. The Picard number of $\bar{M}_{2,1}$ is three, and $B_{3}$ is contracted by $p$.

In terms of the ordered basis $\left(\Delta_{i r r}, \Delta_{1}, W\right)$ it is easy to check (using the $n=7$ example of 88 ) that the pushforward of $\operatorname{Nem}\left(X_{7,1}\right)$ to $\bar{M}_{2,1}$ is generated by $A:=$ $(1,1,0), B:=(1,6,0), D:=(1,6,20)$, and $E:=(3,3,10)$. In fact,

10.2. Proposition. $\operatorname{Mov}\left(\bar{M}_{2,1}\right)=p_{*} \operatorname{Nem}\left(X_{7,1}\right)=\mathbb{R}^{\geq 0} A+\mathbb{R}^{\geq 0} B+\mathbb{R}^{\geq 0} D+\mathbb{R}^{\geq 0} E$.

Proof. By Proposition 2.4, it suffices to show $A, B, D$, and $E$ are generated by moving linear systems. From Lemma 2.3 and Remark (b) following the $n=7$ example of $\sqrt{8}$, the divisor $D$ moves, since it has a moving preimage in $X_{7,1}$. The classes $A$ and $B$ correspond to the classes $\lambda$ and $12 \lambda-\delta_{i r r}$ of $\bar{M}_{2}$, respectively, so both are basepoint free (the characteristic $p$ case follows from the results of Kee99]). $E$ moves since it can be written as an effective combination of either $W$ and $A$ or $\Delta_{i r r}$ and $D$.

Remarks. (1) $\operatorname{Nem}\left(\bar{M}_{2,1}\right)$ is also the cone of Proposition 10.2, as can be directly calculated [Rul01].

(2) The pushforward of $\operatorname{Nef}^{1}\left(X_{7,1}\right)$ (presented in the $n=7$ example of 98 ) is the convex hull of the rays generated by $A, B$, and $D$.

(3) The equations (17) give

$$
30 p^{*} \omega \equiv(5,12,6,2) .
$$

Recalling that $b_{5}:=\frac{1}{2} B_{5}$ and using the fact that $p$ has connected fibres,

$$
C:=30 \omega=30 p_{*} p^{*} \omega \equiv\left(\Delta_{i r r}+6 \Delta_{1}+5 W\right) .
$$

$\operatorname{Nef}^{1}\left(\bar{M}_{2,1}\right)=\mathbb{R}^{\geq 0} A+\mathbb{R}^{\geq 0} B+\mathbb{R}^{\geq 0} C$ is easily calculable using test curves and the facts that $A, B$, and $C$ are nef. $\left(N e f^{1}\left(\bar{M}_{2,1}\right)\right.$ is furthermore the pull-back of $N e f^{1}\left(\bar{M}_{3}\right)$ under the morphism induced by $\Delta_{1} \subseteq \bar{M}_{3}$ [Rul01].) 
(4) The class $\omega$ of $\bar{M}_{2,1}$ is semi-ample in any characteristic (the char $p$ case follows from the results of [Kee99]; the char 0 case is shown in [Rul01]), so every line bundle in $\operatorname{Nef}^{1}\left(\bar{M}_{2,1}\right)$ is eventually free.

(5) The inequalities of Corollary 9.2 cut out the nef cone of $\bar{M}_{2,1}$; i.e. any effective divisor on $\bar{M}_{2,1}$ pulling back to a member of $\operatorname{Nem}\left(X_{7,1}\right)$ is nef.

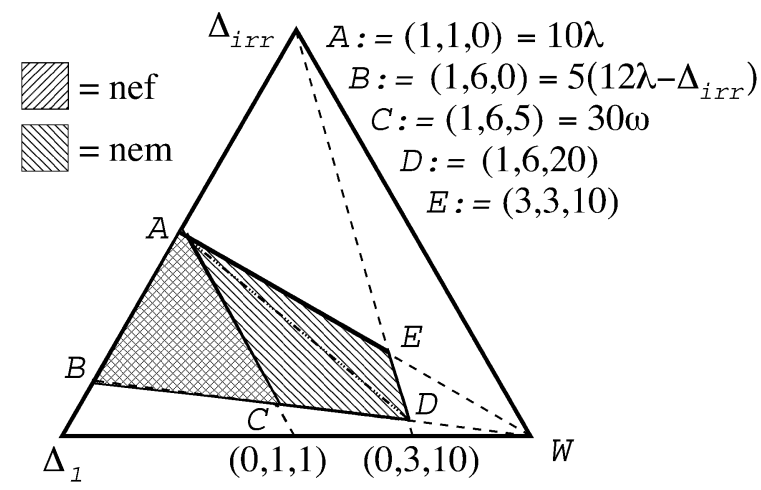

FiguRE 8. A cross section of $\overline{N E}^{1}\left(\bar{M}_{2,1}\right)$.

We partially decompose $\operatorname{Mov}\left(\bar{M}_{2,1}\right)$, in characteristic zero.

10.3. Proposition (char 0 ). $X_{7,1}$ resolves a rational map $\bar{M}_{2,1} \rightarrow Z$, where $Z$ is $a \mathbb{Q}$-factorial projective variety isomorphic to $\bar{M}_{2,1}$ in codimension one. The nef cone of $Z$ is identified with the convex hull of the divisors $A, C$, and D (Figure 8).

Proof. The morphism $p: X_{7,1} \rightarrow \bar{M}_{2,1}$ contracts the curve class

$$
C_{1} \equiv \check{b}_{5}+2 \check{b}_{2}-\check{b}_{3}
$$

which is obtained from a general member of $B_{3}$ by varying the node along the component containing the distinguished marked point. Since the relative Picard number of $p$ is one, $C_{1}$ is the only curve class contracted by $p$; since $B_{3}$ intersects $C_{1}$ negatively, any curve numerically equivalent to $C_{1}$ is contained in $B_{3}$, so $p$ is an isomorphism outside of $B_{3}$.

Let $C_{2}$ be the curve class of $X_{7,1}$ obtained by varying the node of a general member of $B_{3}$ along the other component, so

$$
C_{2} \equiv 4 \check{b}_{4}-2 \check{b}_{3}
$$

Intersection with $C_{2}$ determines a supporting hyperplane of $N e f^{1}\left(X_{7,1}\right)$, and $C_{2}^{\perp} \cap$ $\operatorname{Nef}^{1}\left(X_{7,1}\right)$ is the convex hull of $(0,2,1,2),(5,12,6,2)$, and $(10,6,3,1)$, so is a fulldimensional face; thus $C_{2}$ is an extremal ray of the Mori cone $\overline{N E}_{1}\left(X_{7,1}\right)$. We will use the Cone Theorem [KM98, Theorem 3.7, p. 76] to get a morphism $q$ of $X_{7,1}$ contracting only the class $C_{2}$.

For $n=7$ Riemann-Hurwitz and [KM96, Lemma 3.5] or [Pan97, Prop. 1] give

$$
K_{X_{7,1}} \equiv-\frac{1}{3} b_{2}-\frac{4}{3} b_{5},
$$

which dots $C_{2}$ trivially. $X_{n, 1}$ is a finite quotient of the smooth variety $X_{n}$, and the boundary of $X_{n}$ is snc, so $\left(X_{7,1}, \frac{1}{2} B_{3}\right)$ is klt. By the Cone Theorem there exists a morphism $q: X_{7,1} \rightarrow Z$ with connected fibres onto a normal, projective variety $Z$, 
contracting only curves numerically equivalent to $C_{2}$ (so $q$ contracts $B_{3}$ and is an isomorphism outside of it). Since the relative Picard number of $q$ is one and $B_{3}$ is contracted, $Z$ is $\mathbb{Q}$-factorial.

Finally, $q^{*} N e f^{1}(Z)$ is the face of $N e f^{1}\left(X_{7,1}\right)$ determined by $C_{2}^{\perp}$, so $p_{*} q^{*} N e f^{1}(Z)$ is the convex hull of the rays generated by $A, C$, and $D$.

\section{REFERENCES}

[DM69] P. Deligne and D. Mumford, The irreducibility of the space of curves of given genus, Inst. Hautes Études Sci. Publ. Math. (1969), no. 36, 75-109. MR0262240 (41:6850)

[Fab99] Carel Faber, Algorithms for computing intersection numbers on moduli spaces of curves, with an application to the class of the locus of Jacobians, New trends in algebraic geometry (Warwick, 1996), London Math. Soc. Lecture Note Ser., vol. 264, Cambridge Univ. Press, Cambridge, 1999, pp. 93-109. MR.1714822 (2000m:14032)

[HK00] Yi Hu and Sean Keel, Mori dream spaces and GIT, Michigan Math. J. 48 (2000), 331348, Dedicated to William Fulton on the occasion of his 60th birthday. MR1786494 (2001i:14059)

[HM98] Joe Harris and Ian Morrison, Moduli of curves, Graduate Texts in Mathematics, vol. 187, Springer-Verlag, New York, 1998. MR1631825 (99g:14031)

[HT02] Brendan Hassett and Yuri Tschinkel, On the effective cone of the moduli space of pointed rational curves, Topology and geometry: commemorating SISTAG, Contemp. Math., vol. 314, Amer. Math. Soc., Providence, RI, 2002, pp. 83-96. MR.1941624 (2004d:14028)

[Kee92] Seán Keel, Intersection theory of moduli space of stable n-pointed curves of genus zero, Trans. Amer. Math. Soc. 330 (1992), no. 2, 545-574. MR1034665 (92f:14003)

[Kee99] _ Basepoint freeness for nef and big line bundles in positive characteristic, Ann. of Math. (2) 149 (1999), no. 1, 253-286. MR1680559 (2000j:14011)

[KM96] Seán Keel and James McKernan, Contractible extremal rays on $\bar{M}_{0, n}$, Preprint, alggeom/9607009, July 1996.

[KM98] János Kollár and Shigefumi Mori, Birational geometry of algebraic varieties, Cambridge Tracts in Mathematics, vol. 134, Cambridge University Press, Cambridge, 1998, With the collaboration of C. H. Clemens and A. Corti, Translated from the 1998 Japanese original. MR 1658959 (2000b:14018)

[Kol96] János Kollár, Rational curves on algebraic varieties, Ergebnisse der Mathematik und ihrer Grenzgebiete. 3. Folge. A Series of Modern Surveys in Mathematics [Results in Mathematics and Related Areas. 3rd Series. A Series of Modern Surveys in Mathematics], vol. 32, Springer-Verlag, Berlin, 1996. MR1440180 (98c:14001)

[Mor01] Atsushi Moriwaki, The $\mathbb{Q}$-Picard group of the moduli space of curves in positive characteristic, Internat. J. Math. 12 (2001), no. 5, 519-534. MR1843864 (2002j:14033)

[Pan97] Rahul Pandharipande, The canonical class of $\bar{M}_{0, n}\left(\mathbf{P}^{r}, d\right)$ and enumerative geometry, Internat. Math. Res. Notices (1997), no. 4, 173-186. MR.1436774 (98h:14067)

[POR] PORTA, POlyhedron Representation Transformation Algorithm, avaliable at http://www.iwr.uni-heidelberg.de/groups/comopt/software/PORTA.

[Rul01] William Rulla, The birational geometry of $\bar{M}_{3}$ and $\bar{M}_{2,1}$, Ph.D. thesis, University of Texas at Austin, 2001.

[Ver02] Peter Vermeire, A counterexample to Fulton's conjecture on $\bar{M}_{0, n}$, J. Algebra 248 (2002), no. 2, 780-784. MR1882122(2002k:14043)

Department of Mathematics, University of Georgia, Athens, Georgia 30602

E-mail address: rulla@math.uga.edu 